\title{
Ralstonia mannitolilytica sepsis: a case report
}

\author{
Michael Owusu 1,2, Godfred Acheampong², Augustina Annann 2,3, Kwadwo Sarfo Marfo², Isaac Osei², John Amuasi ${ }^{2,4}$, \\ Nimako Sarpong ${ }^{5}$, Justin Im $^{6}$, Ondari D. Mogeni ${ }^{6}$, Hsin-Ying Chiang ${ }^{7}$, Chih-Horng Kuo ${ }^{7}$, Hyon Jin Jeon ${ }^{6}$, \\ Ursula Panzner ${ }^{6}$, Se Eun Park ${ }^{6}$, Florian Marks ${ }^{6}$, Ellis Owusu-Dabo ${ }^{2,4^{*}}$ and Yaw Adu-Sarkodie ${ }^{8}$
}

\begin{abstract}
Background: Ralstonia mannitolilytica is an emerging opportunistic pathogen that is associated with severe disease, including septic shock, meningitis, and renal transplant infections. Reports on this pathogen are limited, however, especially on the African continent.

Case presentation: A 2-year-old Akan child was presented to a hospital in the northeastern part of Ghana with a 1-week history of fever and chills. We identified Ralstonia mannitolilytica in her blood culture using both conventional and $16 \mathrm{~S}$ ribosomal deoxyribonucleic acid (rDNA) techniques. The patient's condition improved clinically upon treatment with cefuroxime.

Conclusion: Our report highlights the potential of Ralstonia mannitolilytica to cause sepsis and thus emphasizes the need for improved laboratory diagnosis and evidence for use of appropriate antibiotics in rural settings of Africa, where presumptive treatment using antimicrobial agents is rife.
\end{abstract}

Keywords: Ralstonia mannitolilytica, Sepsis, Nonfermenting gram-negative rods, Case report, $16 \mathrm{~S}$ rRNA

\section{Background}

Nonfermenting gram-negative rods are one of the commonest causes of nosocomial infections in clinical environments. The major opportunistic pathogens in this group are Acinetobacter baumanii; Stenotrophomonas maltophilia; and other oxidase-positive bacteria, such as Pseudomonas aeruginosa and Burkholderia cepacia.

Ralstonia mannitolilytica ( $R$. mannitolilytica) is another emerging opportunistic pathogen within the nonfermenting gram-negative bacillus group that is present in both hospital and environmental settings [1]. R. mannitolilytica had previously been referred to as "Pseudomonas thomasii" and $R$. pickettii biovar 3/“thomasii" [2]. Although clinical infections with this pathogen are rare, disease progression to severity tends to be more serious once individuals are exposed. A large oncology hospital in Rome recently reported R. mannitolilytica infections among 12 oncology

\footnotetext{
* Correspondence: owusudabo@kccr.de; owusudabo@yahoo.com

${ }^{2}$ Kumasi Centre for Collaborative Research in Tropical Medicine, Kwame

Nkrumah University of Science and Technology, Kumasi, Ghana

${ }^{4}$ Department of Global Health, School of Public Health, Kwame Nkrumah

University of Science and Technology, Kumasi, Ghana

Full list of author information is available at the end of the article
}

outpatients attending a day ward [3]. China similarly reported three cases of bloodstream infections with $R$. mannitolilytica [4], and Belgium recorded two clinical cases of recurrent meningitis on an implanted intraventricular catheter and an infected hemoperitoneum [5].

Although these infections could potentially occur in Africa, especially as a result of poor environmental conditions and infection prevention control practices, reports on them are rare, perhaps because of limited diagnostic capacity. We report a case of $R$. mannitolilytica sepsis in a 2-year-old child at a rural hospital in the Ashanti Region in Ghana.

\section{Case presentation}

A 2-year-old Akan child was presented to a rural hospital in the Ashanti Region of Ghana with a 1-week history of fever that had been controlled with the use of acetaminophen syrup. On examination, the patient weighed $19.0 \mathrm{~kg}$, was anicteric, and looked pale. Her heart rate was 132 beats per minute with normal heart sounds. Results of her respiratory and abdominal examinations were also normal. On the basis of clinical findings, provisional diagnoses of 
malaria and sepsis were made. Pending laboratory results, she was empirically treated with $650 \mathrm{mg}$ of intravenous cefuroxime three times daily and $50 \mathrm{mg}$ of intravenous artesunate at 4-hourly intervals.

Her full blood count investigation showed a hemoglobin concentration of $9.2 \mathrm{~g} / \mathrm{dl}$, total white blood cell count of $5.6 \times 10^{3}$ cells $/ \mu$ l, and platelet count of $81 \times 10^{9}$ cells $/ \mu$ l. The result of her malaria smear test was positive.

Urine and stool culture results were negative for any bacteria. The blood culture, however, yielded a nonfermenting gram-negative bacterium. The bacterium was processed for identification using the analytic profile index (API) (bioMérieux, Marcy-l'Étoile, France) specific to non-Enterobact eriaceae (API-20NE). This showed an Identification number (ID) of 0045477, which was consistent with $R$. pickettii. Further confirmation was achieved using the $16 \mathrm{~S}$ ribosomal ribonucleic acid (rRNA) method described in our previous study [6]. In summary, deoxyribonucleic acid (DNA) was extracted from pure culture of the bacterium using the
SpheroLyse extraction kit (Hain Lifesciense GmbH, Nehren, Germany). The $16 \mathrm{~S}$ rDNA was amplified using primer pair $8 \mathrm{~F}$ and $1492 \mathrm{R}$, and the resulting sequence was checked using DECIPHER (version 2.2.0). On the basis of the BLASTN sequence similarity search against the NCBI $16 \mathrm{~S}$ rRNA sequence database, the strain was found to be the strain type of $R$. mannitolilytica $(1350 / 1360=99.3 \%$ sequence identity) (Fig. 1). The sequence has been deposited in the National Center for Biotechnology Information database [GenBank:MF590120].

Antimicrobial susceptibility testing of the isolate showed resistance to ampicillin and sensitivity to gentamicin, cefuroxime, ciprofloxacin, ceftriaxone, and cotrimoxazole. The patient's condition improved clinically upon treatment with cefuroxime.

\section{Discussion and conclusion}

R. mannitolilytica infections are not common in clinical settings. The few reported cases have been of the sister

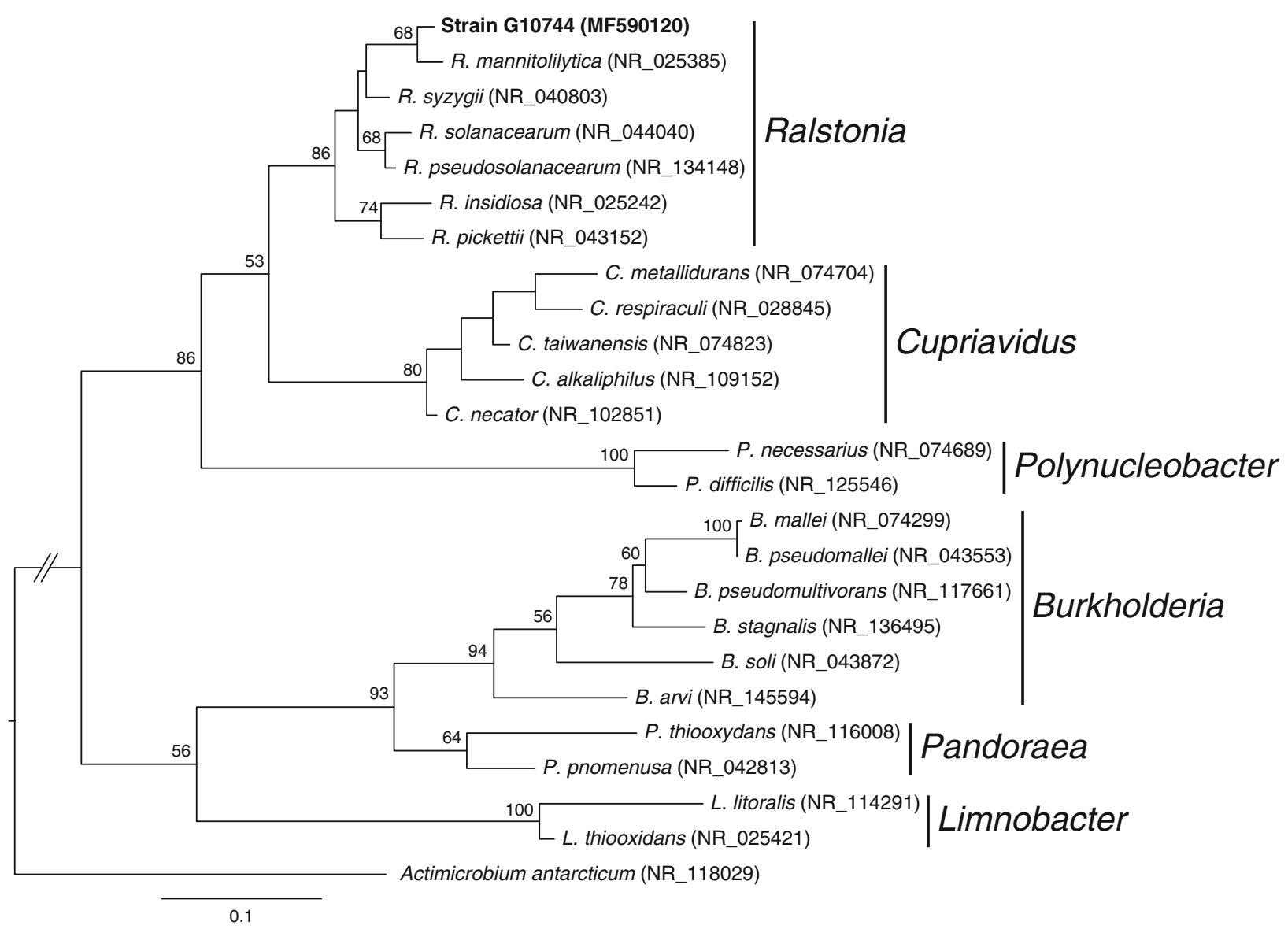

Fig. 1 Maximum likelihood phylogeny based on 16S rRNA gene sequences. The GenBank accession number for each sequence is provided in parentheses next to the species name abbreviation The genus name is listed to the right of all applicable entries. The levels of bootstrap support based on 1000 replications are labeled above internal branches; only values greater than $50 \%$ are listed. On the basis of a BLASTN sequence similarity search against the NCBI 165 rRNA sequence database, the strain G10744 is most similar to the type strain of Ralstonia mannitolilytica $(1350 / 1360=$ 99.3\% sequence identity). Other representative species from the family Burkholderiaceae are included to infer the phylogenetic placement of G10744. The sequence from Actimicrobium antarcticum (family Oxalobacteraceae) is included as the outgroup to root the phylogeny 
genus, $R$. pickettii. Although less frequent, the few documented cases are severe, with reported incidences in hospital outbreaks, bacteremia and bacteriuria, meningitis, renal transplant infection, and hemoperitoneum infection $[4,5,7]$. In rare cases, $R$. mannitolilytica has been isolated from contaminated oxygen delivery devices [1]. Our patient was a 2-year-old child residing in a rural part of Ghana. She had no known history of any underlying chronic disease or immunological suppression apart from her presentation of fever and chills. The presence of this bacterium in the blood is of importance, being the first such report from West Africa. Although it might be difficult to determine the extent of disease severity on account of malaria or sepsis, the isolation of a pure strain of the bacterium in blood suggests a pathogenic association with sepsis. Our report is not different from a recent review of three infants from China who similarly presented with chills and fever [4]. Their cases progressed to septic shock, however, with symptoms including increased heart rate and decreased urine output. We also observed reduced sensitivity in the accuracy of speciation by the API. Although the API identified this bacterium as $R$. pickettii, the $16 \mathrm{~S}$ rDNA technique revealed this as $R$. mannitolilytica. Other authors have similarly reported variations and inconsistencies in the use of standard biochemistrybased techniques for identification of Ralstonia because these techniques share similar biochemical patterns [8]. This emphasizes the importance of using molecular techniques as a supporting diagnostic confirmation, especially for nonfermenting bacteria.

Antimicrobial susceptibility testing also showed susceptibility to antibiotics, including gentamicin, cefuroxime, ciprofloxacin, ceftriaxone, and cotrimoxazole. Treatment and management of Ralstonia spp. has been reported as challenging because of their intrinsic resistance to inducible $\beta$-lactamases [9]. The isolated bacterium did not exhibit this form of resistance. A more structured epidemiological study would be helpful to further evaluate this occurrence.

This report shows that $R$. mannitolilytica might be more widely distributed than previously thought. Active surveillance is therefore recommended to further understand its epidemiology, public health impact, and geographic distribution.

\section{Abbreviation \\ API: Analytic profile index}

\section{Acknowledgements}

We thank the parents of the child and the staff of the hospital. The Sanger sequencing service was provided by the DNA Analysis Core Laboratory (Institute of Plant and Microbial Biology, Academia Sinica, Taipei, Taiwan).

\section{Authors' contributions}

MO, FM, EOD, and YAS designed the study and contributed to the writing of the manuscript. KSM, AA, UP, and GA contributed to routine laboratory analysis of the samples. IO, JA, NS, JI, ODM, SEP and HJJ contributed to data collection, documentation of clinical findings, and the writing of the manuscript. HYC and CHK assisted with sequencing, sequence data interpretation, and the writing of the manuscript. All authors read and approved the final manuscript.

\section{Funding}

This report describes a subject recruited as part of an ongoing study that is a partnership between the Kumasi Centre for Collaborative Research based at the Kwame Nkrumah University of Science and Technology, Kumasi, Ghana, and the International Vaccine Institute, Seoul, Republic of Korea, with sponsorship from the Bill and Melinda Gates Foundation.

\section{Availability of data and materials}

All data generated or analyzed during this study are included in this published article, and the sequences have been deposited in the GenBank database.

\section{Ethics approval and consent to participate}

This report was generated as part of a study that is investigating the etiological agents of sepsis in Ghana. The study has been approved by the Committee of Human Research, Ethics and Publication of the Kwame Nkrumah University of Science and Technology.

\section{Consent for publication}

Written informed consent was obtained from the patient's legal guardians for publication of this case report and any accompanying images. A copy of the written consent is available for review by the Editor-in-Chief of this journal.

\section{Competing interests}

The authors declare that they have no competing interests.

\section{Author details}

'Department of Medical Diagnostics, Kwame Nkrumah University of Science and Technology, Kumasi, Ghana. ${ }^{2}$ Kumasi Centre for Collaborative Research in Tropical Medicine, Kwame Nkrumah University of Science and Technology, Kumasi, Ghana. ${ }^{3}$ Department of Theoretical and Applied Biology, Kwame Nkrumah University of Science and Technology, Kumasi, Ghana. ${ }^{4}$ Department of Global Health, School of Public Health, Kwame Nkrumah University of Science and Technology, Kumasi, Ghana. ${ }^{5}$ Agogo Presbyterian Hospital, Agogo, Ashanti Region, Ghana. ${ }^{6}$ Department of Epidemiology, International Vaccine Institute, Seoul, Republic of Korea. ${ }^{7}$ Institute of Plant and Microbial Biology, Academia Sinica, Taipei, Taiwan. ${ }^{8}$ Department of Clinical Microbiology, Kwame Nkrumah University of Science and Technology, Kumasi, Ghana.

Received: 22 April 2019 Accepted: 16 August 2019

Published online: 26 October 2019

\section{References}

1. Jhung MA, Sunenshine RH, Noble-Wang J, Coffin SE, St John K, Lewis FM, Jensen B, Peterson A, LiPuma J, Arduino MJ, et al. A national outbreak of Ralstonia mannitolilytica associated with use of a contaminated oxygendelivery device among pediatric patients. Pediatrics. 2007;119:1061-8.

2. De Baere T, Steyaert S, Wauters G, Des Vos P, Goris J, Coenye T, Suyama T, Verschraegen G, Vaneechoutte M. Classification of Ralstonia pickettii biovar 3/'thomasii' strains (Pickett 1994) and of new isolates related to nosocomial recurrent meningitis as Ralstonia mannitolytica sp. nov. Int J Syst Evol Microbiol. 2001;51:547-58.

3. Lucarelli C, Di Domenico EG, Toma L, Bracco D, Prignano G, Fortunati M, Pelagalli L, Ensoli F, Pezzotti P, García-Fernández A, et al. Ralstonia mannitolilytica infections in an oncologic day ward: description of a cluster among high-risk patients. Antimicrob Resist Infect Control. 2017;6:20.

4. Liu CX, Yan C, Zhang P, Li FQ, Yang JH, Li XY. Ralstonia mannitolilyticainduced septicemia and homology analysis in infected patients: 3 case reports. Jundishapur J Microbiol. 2016;9:e34373.

5. Vaneechoutte M, De Baere T, Wauters G, Steyaert S, Claeys G, Vogelaers D, Verschraegen $G$. One case each of recurrent meningitis and hemoperitoneum infection with Ralstonia mannitolilytica. J Clin Microbiol. 2001;39:4588-90.

6. Owusu M, Owusu-Dabo E, Acheampong G, Osei I, Amuasi J, Sarpong N, Annan A, Chiang HY, Kuo CH, Park SE, et al. Pseudomonas oryzihabitans sepsis in a 1-year-old child with multiple skin rashes: a case report. J Med Case Rep. 2017;11:77. 
7. Mukhopadhyay C, Bhargava A, Ayyagari A. Ralstonia Mannitolilytica infection in renal transplant recipient: first report. Indian J Med Microbiol. 2003;21: 284-6.

8. Coenye T, Spilker T, Reik R, Vandamme P, Lipuma JJ. Use of PCR analyses to define the distribution of Ralstonia species recovered from patients with cystic fibrosis. J Clin Microbiol. 2005:43:3463-6.

9. Girlich D, Naas T, Nordmann P. OXA-60, a chromosomal, inducible, and imipenem-hydrolyzing class $D$ B-lactamase from Ralstonia pickettii. Antimicrob Agents Chemother. 2004;48:4217-25.

\section{Publisher's Note}

Springer Nature remains neutral with regard to jurisdictional claims in published maps and institutional affiliations.

Ready to submit your research? Choose BMC and benefit from:

- fast, convenient online submission

- thorough peer review by experienced researchers in your field

- rapid publication on acceptance

- support for research data, including large and complex data types

- gold Open Access which fosters wider collaboration and increased citations

- maximum visibility for your research: over $100 \mathrm{M}$ website views per year

At BMC, research is always in progress.

Learn more biomedcentral.com/submissions 